\title{
REVIEW
}

\section{Implications of the new definition of myocardial infarction}

\section{C-K Wong, H D White}

Postgrad Med J 2005;81:552-555. doi: 10.1136/pgmi.2005.035071

The new definition of acute myocardial infarction is based primarily on raised troponin levels because of the sensitivity and specificity of these markers and their correlation with the pathophysiology of acute coronary syndromes with plaque fissuring or rupture and embolisation of platelets causing myocyte necrosis. Raised troponin levels are associated with increased risks of death and recurrent myocardial infarction. Greater treatment benefit with low molecular weight heparin, $\mathrm{Ilb} / \mathrm{llla}$ antagonists and revascularisation is seen when troponin levels are raised. There are many implications for patients and society of the new definition including changes in insurability and ability to continue certain occupations. Many more patients, who would previously been diagnosed as having unstable angina, will now be diagnosed as having had an acute myocardial infarction. In addition case fatality rates will fall and comparison with previous epidemiological studies using the old definition will be problematic. However, the new definition may result in greater use of evidence based therapies with improved patient outcomes and decreased community death rates.

\begin{abstract}
See end of article for authors' affiliations

Correspondence to: Professor H D White, Green Lane Cardiovascular Service Auckland City Hospital Private Bag 92189 Auckland 1030, New Zealand; harveyw@adhb. govt.nz
\end{abstract}

Submitted 15 March 2005 Accepted 15 March 2005

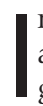
n 2000, the European Society of Cardiology and American College of Cardiology promulgated a new definition of myocardial infarction, which is summarised in box $1 .{ }^{1}$ For the acute setting, the definition is based mainly on biochemical evidence of myocardial necrosis (rising and falling levels of cardio-specific biomarkers preferably troponin $\mathrm{T}$ or I), in an appropriate context of a clinical acute coronary syndrome or post-percutaneous coronary intervention (PCI).

A salient point in this re-definition is the emphasis of a cardio-specific biomarker whereas in the original World Health Organisation definition a combination of symptoms and classic ECG changes was sufficient for diagnosis. ${ }^{2}$ The European Society of Cardiology and American College of Cardiology Committee stated the precision requirement for the biomarker to reflect myocardial necrosis (>99th centile of the normal reference population and with high reproducibility - that is, a coefficient of variation of below 10\%), and introduced the concept that any increase in the marker reflecting any amount of necrosis is regarded as an acute myocardial infarction (AMI) provided the clinical context is compatible. ${ }^{1}$ The committee also recommended blood sampling at presentation, six to nine hours later, and if clinically appropriate repeated at 12-24 hours. ${ }^{1}$

As the European Society of Cardiology and American College of Cardiology Committee acknowledged, ${ }^{1}$ with the new definition and the increasing availability of highly sensitive cardiac marker analysis, more patients (perhaps double from $20 \%$ to $45 \%)^{3}$ would be diagnosed as having an AMI when they present with a non-ST elevation acute coronary syndrome whereas hitherto they were labelled as having unstable angina. This will consequently lead to an increasing number of AMI diagnoses with a decrease in the case fatality rate. The implications of this re-definition are important and affects several areas.

(1) Epidemiology. A transition period will be required for epidemiologists to compare the new definition of myocardial infarction (MI) with the traditional WHO definition.

(2) Clinical trials. MI is often an inclusion criterion for trials and with the new definition the infarcts will be smaller. MI is also often an end point in clinical trials and with the new definition there will be many more end points than previously perhaps carrying less prognostic significance. Assessment of the effectiveness of new therapies and translation into clinical practice will also be more difficult than in the past.

(3) The natural history and evolution of coronary disease in individual patients is now more likely to be punctuated with multiple episodes of AMIs.

(4) Social and public policy including insurability, ability to work, and funding.

\section{USE OF TROPONIN ASSAYS}

The cardiac troponin assays are more sensitive and specific than CK or CKMB assays. Current assays for cardiac troponins use monoclonal antibodies that specifically bind to cardiac but not skeletal forms of troponins. It takes about six to eight hours for troponin levels to become raised after the onset of myocardial ischaemia. The test is highly sensitive to even minute amounts of troponins and it is not clear whether small leaks from the cytosol without necrosis of the myocardial contractile apparatus (made up of troponin T, I, and C) may be detected by the current assays. However, troponins are large proteins and for detection in the blood the cell membrane has to be very leaky and probably

Abbreviations: $\mathrm{Ml}$, myocardial infarction; $\mathrm{AMl}$, acute myocardial infarction; $\mathrm{PCl}$, percutaneous coronary intervention 
Box 1 Definition of myocardial infarction based on the European Society of Cardiology and American College Of Cardiology consensus statement

Criteria for acute, evolving, or recent myocardial infarction

Either one of the following criteria satisfies the diagnosis for an acute, evolving, or recent myocardial infarction:

- Typical rise and gradual fall (troponin) or more rapid rise and fall (CKMB) of biochemical markers of myocardial necrosis with at least one of the following:

- ischaemic symptoms;

- development of pathological Q waves on the ECG;

- ECG changes indicative of ischaemia (ST segment elevation or depression); or coronary artery intervention (for example, coronary angioplasty).

- Abnormal findings of an acute myocardial infarction

Criteria for established myocardial infarction

Any one of the following criteria satisfies the diagnosis for established myocardial infarction:

- Development of new pathological $Q$ waves on serial ECGs. The patient may or may not remember previous symptoms. Biochemical markers of myocardial necrosis may have normalised, depending on the length of time that has passed since the infarct developed.

- Abnormal findings of a healed or healing myocardial infarction.

irreversibly damaged. Current third generation troponin $\mathrm{T}$ tests use recombinant human cardiac troponin $\mathrm{T}$ for standardisations. Because of patent issues, only a single manufacturer (Roche Diagnostics, formerly Boehringer Mannheim of Mannheim, Germany) has produced a troponin $\mathrm{T}$ assay that has a comparatively uniform cut off concentration and a high precision at this lower end of the measurement range. There is also a troponin $\mathrm{T}$ point of care test. In contrast, there are about 20 different cardiac troponin I assays and inter-assay and intra-assay variability differs depending on the sensitivity and specificity of the immunoassay.

In patients with a non-ST elevation acute coronary syndrome, increase in troponin levels is associated with an almost 10-fold increase in the risk of death or MI in the next 30 days. ${ }^{4}$ This increased risk is not only attributable to the amount of myonecrosis (higher troponin levels being associated with higher mortality $)^{5}$ but also to the association with the presence of coronary thrombus and complex coronary artery stenoses in these patients ${ }^{67}$ with microembolism of platelets and fibrin causing myocyte necrosis (fig 1). ${ }^{7}$ Glycoprotein IIb/IIIa inhibitors, ${ }^{8}$ low molecular weight heparins, ${ }^{9}$ and an early invasive strategy ${ }^{10}$ have all been shown to have greater benefit in troponin positive patients than in troponin negative patients.

\section{POST-PCI MYOCARDIAL INFARCTION}

The European Society of Cardiology and American College of Cardiology Committee acknowledged that myocardial cell injury after PCI is often a single event, in contrast with the often repetitive nature of spontaneously occurring episode of myocardial ischaemia and necrosis in patients with acute coronary syndromes. ${ }^{1}$ However these patients are at higher risk than those without increased markers. ${ }^{1}$ Data from the Cleveland Clinic Foundation Interventional Registry, involving 3478 patients with PCI and with coronary stenting, 15\% with graft intervention and $61 \%$ with use of glycoprotein IIb/ IIIa inhibitors showed that any increase in CKMB was associated with adverse outcome including MI and sudden death over a 15 month follow up period. ${ }^{11}$ The gradient of mortality risk from an increasing level of CKMB has been shown to be similar with PCI and with acute coronary syndromes, suggesting that even minor increases in cardiac markers after PCI are prognostically important. ${ }^{12}$ Increase in troponin levels after PCI has also been shown to increase the risk for death and MI. ${ }^{13}$

\section{UNRESOLVED ISSUES WITH THE NEW DEFINITION}

In the early period after the publication of the consensus statement, several commentators expressed reservations with the new definition, ${ }^{14-17}$ particularly in relation to the impact on epidemiological studies with respect to comparison with previous studies and patients dying from MI in the first few hours in the community or soon after admission and not having had the opportunity for troponin measurements to be made. Concern was also expressed about other causes of an
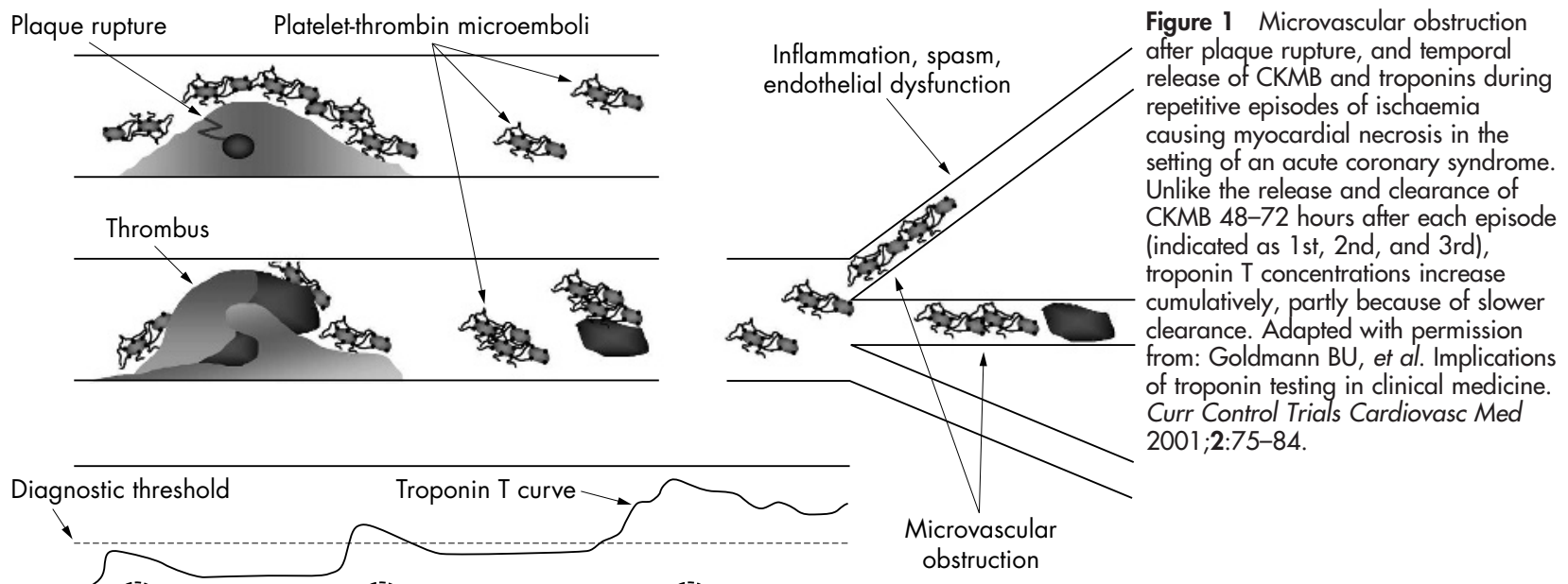

Figure 1 Microvascular obstruction after plaque rupture, and temporal release of $C K M B$ and troponins during repetitive episodes of ischaemia causing myocardial necrosis in the setting of an acute coronary syndrome. the release and clearance of (indicated as 1st, 2nd, and 3rd), tronin $T$ concentrations increase cumulatively, partly because of slowe from: Goldmann BU, et al. Implications of troponin testing in clinical medicine. Curr Control Trials Cardiovasc Med

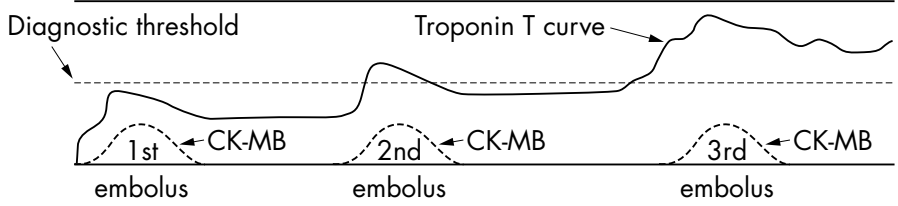
obstruction 
increase in troponin levels and patients with significant renal impairment. In addition there are important implications world wide of a new definition where using a troponin standard may not be possible for cost reasons. ${ }^{18}$

\section{OTHER CAUSES OF RAISED CARDIAC TROPONINS}

Box 2 summarises the various causes of raised troponins in clinical settings other than acute coronary syndromes and PCI. ${ }^{19}$ Troponin levels may increase in patients with heart failure because of raised end diastolic pressures, hormonal effects, and endocardial ischaemia, in patients with ventricular or supraventricular tachyarrhythmias and hypotension, and in patients with acute pulmonary embolism and right ventricular infarction. Myocarditis from various causes is also associated with increased troponin levels.

Thus, while troponins are biomarkers for myocyte necrosis it is critical for physicians to determine the clinical setting of the increased troponins by checking the history, physical examination, and other findings as the basis for the appropriate prognosis, diagnosis, and management. If troponins are not raised it does not mean that patients are at no risk. Patients presenting with non-ST elevation acute coronary syndrome and without an increase in troponin levels have an approximate $4 \%$ incidence of death and MI at 30 days. ${ }^{8}$ Also patients should not be diagnosed as having non-ischaemic chest pain on the basis of having no increase in troponin levels.

\section{RAISED TROPONINS AND RENAL FAILURE}

In renal failure, troponin increase may be attributable to a number of reasons including ischaemic myonecrosis because of concomitant coronary disease or the cardiotoxic effects of raised urea levels. ${ }^{20}$ In the setting of an acute coronary syndrome, troponin $\mathrm{T}$ increase has been shown (using the current generation assay) to have the same negative prognostic prediction for death and MI at 30 days across the entire range of renal function. ${ }^{21}$ Also in clinically stable patients with end stage renal disease on intermittent haemodialysis raised troponin $\mathrm{T}$ levels were seen in 53\% of patients and were independently associated with a several fold increase in two year mortality. ${ }^{22}$ Thus, raised troponin levels have a negative prognostic impact in patients with end stage renal failure, even if there is no clinical indication of coronary disease and may show the need to be more aggressive with anti-atherosclerotic treatments in these patients.

\section{RECOMMENDATIONS FOR EVERYDAY PRACTICE}

(1) The biochemical evidence of troponin increase points to cardiac myocyte necrosis but clinical judgement is required as to whether the rise is attributable to MI related to an acute coronary syndrome or alternatively could be attributable to a non-coronary cause (box 2)

(2) While the troponin standard is most useful in patients with non-ST elevation acute coronary syndrome, patients with ST elevation and presumed new onset left BBB should be managed with reperfusion regardless of the initial troponin level.

(3) With atypical or late presentations of AMI, or in situations where the timing of the MI is not entirely clear, creatine kinase or CKMB is recommended to determine if the event happened in the past 48 hours. Increased troponins can persist in the circulation for up to 14 days, and the serial profile of CKMB is more useful to diagnose re-infarction. Thus laboratories require several markers with cost implications of doing this.

(4) The societal implications of a new definition of MI are profound as more patients will be diagnosed as having MI and this will have an affect on insurability and ability to perform certain occupations such as airline pilots, firemen, and taxi drivers. However evidence based therapy may be used more intensively in these patients with potential community reductions in the morbidity and mortality from coronary artery disease.

(5) The diagnosis of MI without quantifying the prognosis with other factors is not sufficient information to relay to a patient and their family as the prognosis will vary much more than in the past.

Box 2 Causes of raised troponin values in clinical settings other than acute coronary syndromes or percutaneous coronary intervention

Ischaemic causes other than plaque fissuring or rupture

- Coronary embolism (red cell or platelet thrombi, vegetation, atrial myxoma, calcification)

- Coronary spasm

- Coronary dissection

- Aortic dissection

- Transplant vasculopathy

Cardiac surgery

- Left ventricular venting

- Inadequate cardioplegia

- Traumatic atrial cannulation

- Manipulation of the heart

- Ischaemia related causes such as conduit or native vessel occlusion

Miscellaneous

- Tachyarrhythmia

- Hypertension

- Congestive heart failure

- Renal failure

- Drug toxicity (for example, adriamycin, 5-fluorouracil, etc)

- Hypothyroidism

- Pulmonary embolism with right ventricular infarction

- Sepsis (including sepsis occurring with shock)

- Transient ischaemic attack, stroke, or subarachnoid haemorrhage

- Pheochromocytoma

- Rhabdomyolysis with myocyte necrosis

Myopericarditis

- Rheumatic fever

- Rheumatoid arthritis

- Systemic vasculitis

- Post-viral

Infiltrative diseases of the myocardium

- Amyloidosis

- Sarcoidosis

Traumatic

- Atrioventricular ablation

- Defibrillation

- Chest wall trauma 
(6) Patients with non-ST elevation acute coronary syndromes and no increase in troponin levels are at lower risk than patients with raised troponins but are not at negligible risk-that is, an approximate risk of $4 \%$ of MI over the next 30 days with a higher risk if they are diabetic, have inducible ischaemia, or have other risk factors.

(7) There are legal implications associated with the diagnosis of MI. In New Zealand, the definition using troponin standard including the post-PCI setting are accepted by the Land Transport Safety Authority that governs driver licensing. Those with a raised troponin level (without increase in creatine kinase or CKMB) after PCI or in a spontaneous acute coronary syndrome setting are classified in the same way as having a MI, and are not allowed to drive a private vehicle for two weeks or a commercial vehicle for four weeks.

The Redefinition of Myocardial Infarction Committee is currently reviewing the recent redefinition of MI, and focusing on issues such as clinical trial definitions, and definitions of infarction with coronary artery bypass grafting. These changes will also have an important impact on patients and the community.

The change in the definition of MI has had an important impact on our clinical practice. It is probable that the redefinition will lead to greater prescribing and adherence with treatments such as aspirin, $\beta$ blockers, ACE inhibitors, statins, and clopidogrel by patients that may have previously been diagnosed as having non-ischaemic chest discomfort and this will lead to improved patient outcomes and decreased community death rates.

\section{Authors' affiliations}

C-K Wong, Dunedin School of Medicine, Dunedin, New Zealand H D White, Green Lane Cardiovascular Service, Auckland City Hospital, Auckland, New Zealand

Funding: none.

Conflicts of interest: none.

\section{REFERENCES}

1 The Joint European Society of Cardiology/American College of Cardiology Committee. Myocardial infarction redefined-a consensus document of the Joint European Society of Cardiology/American College of Cardiology Committee for the Redefinition of Myocardial Infarction. J Am Coll Cardiol 2000;36:959-69.
2 World Health Organisation Expert Committee. Hypertension and coronary heart disease: classification and criteria for epidemiological studies. Technical Report Series no 168. Geneva: World Health Organisation, 1959.

3 Kontos MC, Fritz LM, Anderson FP, et al. Impact of the troponin standard on the prevalence of acute myocardial infarction. Am Heart J 2003; 146:446-52.

4 Ottani F, Galvani M, Nicolini FA, et al. Elevated cardiac troponin levels predict the risk of adverse outcome in patients with acute coronary syndromes. Am Heart J 2000; 140:917-27.

5 Antman EM, Tanasijevic MJ, Thompson B, et al. Cardiac-specific troponin I levels to predict the risk of mortality in patients with acute coronary syndromes. $N$ Engl $J$ Med 1996;335:1342-9.

6 Lindahl B, Diderholm E, Lagerqvist B, et al. Mechanisms behind the prognostic value of troponin T in unstable coronary artery disease: a FRISC II substudy. J Am Coll Cardiol 2001;38:979-86.

7 White HD. Non-ST-elevation acute coronary syndromes: unstable angina and non-ST-elevation myocardial infarction. In: Topol EJ, ed. Textbook of cardiovascular medicine. Philadelphia: Lippincott Williams and Wilkins, 2002:351-84.

8 Heeschen C, Hamm CW, Goldmann B, et al. Troponin concentrations for stratification of patients with acute coronary syndromes in relation to therapeutic efficacy of tirofiban. Lancet 1999:354:1757-62.

9 Morrow DA, Antman EM, Tanasijevic M, et al. Cardiac troponin I for stratification of early outcomes and the efficacy of enoxaparin in unstable angina: a TIMI-11B substudy. J Am Coll Cardiol 2000;36:1812-17.

10 Morrow DA Cannon CP, Rifai N, et al. Ability of minor elevations of troponins I or T to predict benefit from an early invasive strategy in patients with unstable angina and non-ST elevation myocardial infarction. JAMA 2001;286:2405-12.

11 Brener SJ, Ellis SG, Schneider J, et al. Frequency and long-term impact of myonecrosis after coronary stenting. Eur Heart J 2002;23:869-76.

12 Akkerhuis KM, Alexander JH, Tardiff BE, et al. Minor myocardial damage and prognosis: are spontaneous and percutaneous coronary interventionrelated events different? Circulation 2002; 105:554-6.

13 Wu AH, Boden WE, McKay RG. Long-term follow-up of patients with increased cardiac troponin concentrations following percutaneous coronary intervention. Am J Cardiol 2002;89:1300-2.

14 Dargie H. Myocardial infarction: redefined or reinvented? Heart 2002;88:1-3.

15 Richards AM, Lainchbury JG, Nicholls MG. Unsatisfactory redefinition of myocardial infarction. Lancet 2001;357:1635-6.

16 Tunstall-Pedoe $\mathrm{H}$. Comment on the ESC/ACC redefinition of myocardial infarction by a consensus dissenter. Eur Heart J 2001;22:613-15.

17 Tunstall-Pedoe $\mathbf{H}$. Redefinition of myocardial infarction by a consensus dissenter. J Am Coll Cardiol 2001;37:1472-3.

18 White HD. Things ain't what they used to be: impact of a new definition of myocardial infarction. Am Heart J 2002;144:933-7.

19 French JK, White HD. Clinical implications of the new definition of myocardial infarction. Heart 2004;90:99-106.

20 Musso P, Cox I, Vidano E, et al. Cardiac troponin elevations in chronic renal failure: prevalence and clinical significance. Clin Biochem 1999;32:125-30.

21 Aviles RJ, Askari AT, Lindahl B, et al. Troponin T levels in patients with acute coronary syndromes, with or without renal dysfunction. N Engl J Med 2002;346:2047-52.

22 Apple FS, Murakami MM, Pearce LA, et al. Predictive value of cardiac troponin I and T for subsequent death in end-stage renal disease. Circulation 2002; 106:2941-5. 